\title{
Comparison of Theoretical and Physical Model Studies of the Responses of Moving Source and Fixed Loop Electromagnetic Exploration Systems
}

\author{
K. DuCKWORTH ${ }^{1}$
}

\begin{abstract}
Theoretical and physical scale model responses for moving source and fixed loop electromagnetic exploration systems are presented for the case of a vertical, thin, plate conductor located first in free-space and then in a conductive host. The results are presented in the form of anomaly index diagrams plotted in complex space. The two modeling approaches show general agreement on the behavior of the current gathering effect that operates when the conductive host is present. Both approaches show an onset of a strong positive effect at higher frequencies in the responses provided by both horizontal coplanar coil systems and by fixed loop systems. Agreement is also shown on an unanticipated negative effect that a conductive host causes in the responses provided by the vertical coplanar coincident coil system. Both modeling approaches demonstrate that the responses provided by fixed loop systems are predominantly caused by the current gathering effect. The extent of this effect for fixed loop systems depends on the size of the transmitter loop with respect to the target and on the depth of the target. The theoretical modeling demonstrates that both moving source and fixed loop systems operated over a target located in a conductive host, provide responses that become almost identical and independent of the conductance of the target at high frequency.
\end{abstract}

Key words: Electromagnetic, exploration, theoretical-models, physical scale-models.

\section{Introduction}

In electromagnetic exploration, diagrams of peak anomaly magnitudes plotted in complex space (i.e., socalled phasor or anomaly index diagrams) provide a convenient and compact means of assembling the data for wide ranges of depth and conductance of target conductors (Hedstrom and Parasnis, 1958; Lowrie and West, 1965; NAir et al., 1968; HANNeSOn and West, 1984; DuCKWorth et al., 2001). The following discussion provides examples of anomaly index diagrams generated by physical scale modeling and corresponding diagrams generated by

\footnotetext{
${ }^{1}$ Department of Geology and Geophysics, University of Calgary, 2500 University Drive N.W., Alberta, Canada. E-mail: Ken.Duckworth@ucalgary.ca, http://WWW.GEO.UCALGARY.CA/ duckwrth/kd.html.
} 
theoretical modeling using commercially available software. The results treat targets that are located first in free-space and then in a conductive host and are intended to demonstrate the effect of the gathering or channeling of current from the host into these conductors (GuPTASARMA and MARU, 1971). As far as possible, the two approaches treat closely similar models within the limitation that only thin conductors can be treated by theoretical algorithms currently available in commercial software. While a range of dip values could have been treated by both methods, the results presented here are confined to vertical, thin conductors. These results cover both moving source and fixed loop EM systems and show some expected and some unexpected effects of a target conductor interacting with a conductive host.

\section{Results}

\section{Horizontal Coplanar Coil Responses}

Anomaly index diagrams for secondary field magnitudes detected by horizontal coplanar coils over a thin, vertical, plate conductor located both in free-space and in a conductive host, are shown in Figure 1. Physical scale model results taken from DuCKWORTH and Krebes (1997a) are shown in Figure 1(a), while the lower three diagrams Figures 1(b), 1(c) and 1(d), were generated by a thin plate model developed by WALKER and WEST (1991) that is employed in the EMIGMA software available from PetRosSeikon. In each diagram the conductive host cases are superimposed on the free-space cases, with the free-space cases being represented by dashed contours. The second dispersion seen at high frequencies in the conductive host responses, can be described as a detachment of the conductive host response from the response of the conductor in free-space. This detachment was first described in results obtained by means of physical scale modeling (DUCKWORTH and KREBES 1997a) and is somewhat unusual. It can be argued that unusual effects in scale model responses can be generated by experimental artifacts that will not be present in the full scale system. The present study is intended to demonstrate that the detachment effect is an inherent part of the physics of the system and not an artifact of either the physical scale modeling approach or of the theoretical approach.

The scale model responses were obtained using a graphite plate model of $819 \mathrm{~S}$ conductance (dimensions $1 \mathrm{~m} \times 0.3 \mathrm{~m} \times 0.013 \mathrm{~m}$ conductivity $6.3 \times 10^{4} \mathrm{~S} / \mathrm{m}$ ), placed first in air and then in a brine of $14.5 \mathrm{~S} / \mathrm{m}$ conductivity $(0.069 \mathrm{ohm}-\mathrm{m})$. The coil separation was $0.2 \mathrm{~m}$ so that for the model depth values shown in Figure 1(a), the range of depth to separation ratio values is from 0.1 to 0.25 . The frequencies indicated on the diagram (Figure 1(a)) are the frequencies that were used in the modeling lab.

The two theory generated diagrams Figures 1(b) and 1(c), cover the depth to separation range 0.1 to 0.3 for a coil separation of $200 \mathrm{~m}$ and in each case the 

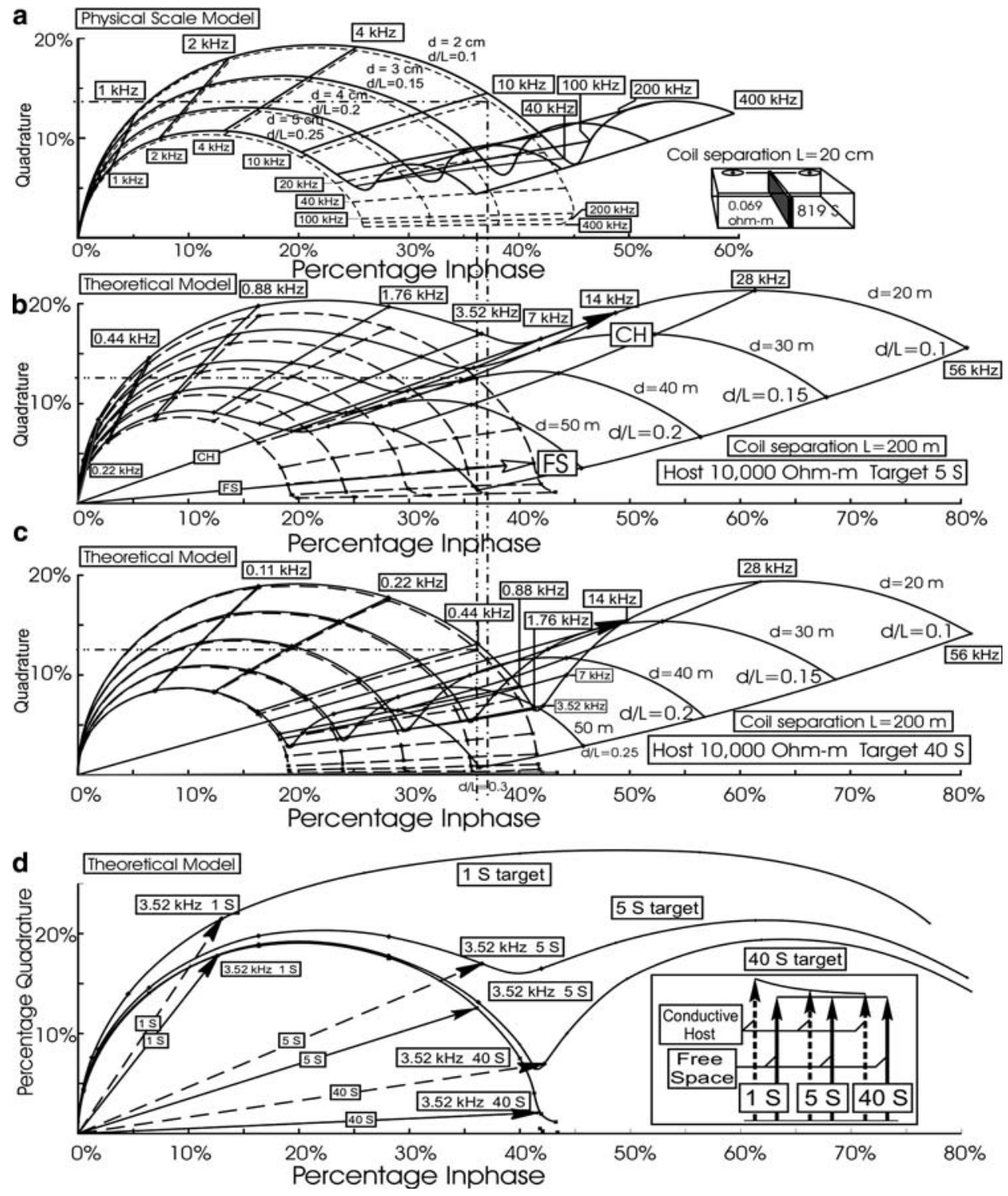

Figure 1

Phasor diagrams derived by physical and theoretical modeling of a Horizontal Coplanar coil system over a vertical plate in free-space and in a conductive host. (a) Physical model: $819 \mathrm{~S}$ graphite plate in a $14.5 \mathrm{~S} / \mathrm{m}$ brine. (b) Theory: $5 \mathrm{~S}$ plate in a $0.0002 \mathrm{~S} / \mathrm{m}(5000 \mathrm{ohm}-\mathrm{m})$ host. (c) Theory: $40 \mathrm{~S}$ plate in a $0.0001 \mathrm{~S} / \mathrm{m}$ $(10000 \mathrm{ohm}-\mathrm{m})$ host. (d) Theory: fixed target depth $(\mathrm{d} / \mathrm{L}=0.1)$ and fixed host conductivity for a range of target conductance values. Inserted box shows that lower conductance targets generate responses that are proportionately more enhanced by current gathering than are the responses of high conductance targets. Vectors CH (for Conductive Host) and FS (Free Space) demonstrate the phase rotation and enhancement at the frequency of $14 \mathrm{kHz}$. In Figures 1(a), 1(b) and 1(c) the conductive host cases (solid lines) are superimposed on the free-space cases, (dashed contours). 
simulated host has a conductivity of $0.0001 \mathrm{~S} / \mathrm{m}$ (i.e. 10,000 ohm-m resistivity). Figures 1(b), and 1(c) treat targets of $5 \mathrm{~S}$ and $40 \mathrm{~S}$ conductance respectively with a strike length of $500 \mathrm{~m}$ and a depth extent of $250 \mathrm{~m}$ for a frequency range from $110 \mathrm{~Hz}$ to $56,000 \mathrm{~Hz}$ such as is available in the Max-Min electromagnetic exploration system.

The physical model results in Figure 1(a) are normalized with respect to the signal recorded when the sensing coils are located in air at sufficient distance from any conductive structure that the response is pure real. The results for the target in free-space shown in Figure 1(a), are in good agreement with earlier published studies (Nair et al. 1968; Strangway, 1966; HanNeSON and West, 1984; FrischKNecht et al., 1991). The physical model system has also been found to generate results that are in good quantitative agreement with theoretical modeling of the response of a cubic structure (FARQUHARSON et al., 2003). The EMIGMA software offers a variety of alternative references for normalization; the option to normalize with respect to "inphase" used in the results presented in Figure 1 provided the closest agreement with the physical model results.

The outstanding feature of agreement between the scale model and theoretical models is the feature described earlier as a detachment of the conductive host response from the free-space response at high frequencies. The most direct comparison is between the scale model results of Figure 1(a) for an $819 \mathrm{~S}$ target in a $0.069 \mathrm{Ohm}-\mathrm{m}$ host and a coil separation of $0.2 \mathrm{~m}$ and the theoretical results in Figure 1(c) for a $40 \mathrm{~S}$ target in a $10,000 \mathrm{ohm}-\mathrm{m}$ host with a coil separation of $200 \mathrm{~m}$. The onset of this detachment at $40 \mathrm{kHz}$ in the scale model data and at $1.76 \mathrm{kHz}$ in the theoretical model data, can be ascribed to the development of strong inductive activity in the host rock and consequent gathering of current into the target from the host. For frequencies above the point of onset of strong current gathering, there is an initial strong anticlockwise phase rotation as well as a marked increase in response magnitude. This is demonstrated in Figure 1(b) by the response vectors labeled $\mathrm{CH}$ (Conductive Host) and FS (Free Space) for $14 \mathrm{kHz}$. Below the frequency at which the strong enhancement begins, the conductive host causes only mild increases of the response magnitude along with anticlockwise phase rotation. The later discussion of vertical coincident coil responses shows the low frequency responses to be more complex than the horizontal coplanar coil responses of Figure 1 suggest.

While both the physical model data and the theoretical data demonstrate the detachment of the conductive host responses from the free-space responses at high frequency, the theoretical results in Figures 1(b) and 1(c) indicate a notably stronger enhancement of the response due to current gathering than does the physical scale model data in Figure 1(a). This might be ascribed to the fact that the strike length of the plate used in the theoretical model and its depth extent were smaller in proportion to the coil separation than was the case for the physical scale model. When the strike length in the theoretical model was increased to $1000 \mathrm{~m}$ and the 
depth extent to $300 \mathrm{~m}$, to more closely approximate the physical model, this caused a markedly poorer fit to the response provided by the physical model. It might be possible to achieve a closer match between the scale model and theoretical responses by making changes in the theoretical model, however the main purpose of the results presented in Figure 1 is to show that the detachment between free-space and conductive host responses occurs in both modes of modeling.

The theoretical responses permit the effects of changes in target conductance to be studied more readily than is the case in physical modeling, and in this regard Figures 1(b) and 1(c) demonstrate some noteworthy features from the exploration point of view. Most notable is the fact that for frequencies above $14 \mathrm{kHz}$ the responses are very similar regardless of the conductance of the target. A 10,000 ohm-m host resistivity - as used in Figures 1(b) and 1(c) - might usually be assumed to have little or no effect on the response of target conductors. Indeed, for frequencies below $3.52 \mathrm{kHz}$ and very high conductance targets, Figure 1(c) indicates that the current gathering effect becomes almost completely detached from the free-space response of the target so that any survey conducted at frequencies below $3.52 \mathrm{kHz}$ would generate responses that would be very close to the free-space response of any high conductance target. By comparison, in that same low frequency range, low conductance targets (Figure 1(b)) will produce responses that are appreciably affected by the current gathering effect. This can be seen in Figure 1(d) where the effect of reducing target conductance from $40 \mathrm{~S}$ to $5 \mathrm{~S}$ and to $1 \mathrm{~S}$ for a fixed target depth of $20 \mathrm{~m}$ (i.e. $\mathrm{d} / \mathrm{L}=0.1$ ) is shown. In Figure 1(d) the freespace and conductive host response vectors for the $40 \mathrm{~S}, 5 \mathrm{~S}$ and $1 \mathrm{~S}$ targets at a frequency of $3.52 \mathrm{kHz}$ are emphasized and are also shown in the insert with the vector magnitudes normalized so that all the free-space vectors are the same size in order that the relatively larger effect on the response of the $1 \mathrm{~S}$ target can be appreciated.

Figure 1(c), for the high conductance $40 \mathrm{~S}$ target, emphasizes the very abrupt onset of strong current gathering for high conductance targets and indicates that given sufficient difference between the response parameter of the target and of its host, the response of the target conductor is able to approach its inductive limit before the host response can move away from its resistive limit; as discussed by Duckworth and Krebes (1997a). The response shown in Figure 1(c) indicates that a survey conducted in the frequency range from $3.52 \mathrm{kHz}$ to $56 \mathrm{kHz}$ over a high conductance target would produce results that would be dominated by the response of the host, yet the interpreter might believe that the response came from the dispersion due to direct induction in the target.

It is also notable in Figure 1(d) that reducing the target conductance to $1 \mathrm{~S}$ causes the separation between the two dispersions (i.e., that due to the target and that due to the host) to become indistinct.

No attempt was made in the physical modeling to obtain responses for a lower conductance target comparable to the $5 \mathrm{~S}$ theoretical responses in Figure 1(b), 
because it would have required machining the graphite to impractically small thicknesses. Any attempt to use higher conductance targets in scale modeling would have required the use of thicker plates of graphite, with the consequence that the target would have no longer responded as a thin plate.

A comparison between the physical and scale model responses may be made by converting the frequency contours to contours of Response Parameter defined as $\alpha=\sigma \mathrm{t} \mu \omega \mathrm{L}$ after HANNESON and WeSt (1984). As an example, the physical scale model response at $10 \mathrm{kHz}$ for the target in free-space with a coil separation of $0.2 \mathrm{~m}$ and a conductance of $819 \mathrm{~S}$ in Figure 1(a) gives an $\alpha$ value of 12.93. The corresponding theoretical response for a $5 \mathrm{~S}$ target and a $200 \mathrm{~m}$ coil separation at $3.52 \mathrm{kHz}$ gives an almost identical response in terms of the magnitudes of the inphase and quadrature components and this gives an $\alpha$ value of 27.79. The difference between these $\alpha$ values can be ascribed to the very nonlinear scale of the complex space type of plot, nonetheless it demonstrates that the two approaches produce results that are comparable.

The theoretical and scale model data both indicate that at the highest frequencies the responses with the conductive host present, approach the inphase axis and will show phase inversion for higher frequencies (or higher host conductivity) particularly for deeper targets. Phase inversion for deeper targets is to be expected because the phase rotation of the fields propagating to and from the target through the conductive host depends on the length of path through the conductive host (LOWRIE and WeSt, 1965; HANNESON and WeSt, 1984).

\section{Vertical Coincident Coil Responses}

Responses obtained for a vertical coincident coil system as shown in Figure 2, illustrate circumstances in which the conductive host can either enhance or diminish the radiated secondary field, depending on the conductivity of the host and on the depth of the target. This contrasts with the responses provided by horizontal coplanar coils (Fig. 1), that show only positive enhancement of the response when a conductive host is present.

The vertical coplanar coincident system (DUCKWORTH et al., 1993) is readily simulated by the EMIGMA software by virtue of its ability to treat the secondary or scattered field separate from the primary transmitted field and to present the results in absolute rather than relative form. Presentation of the secondary field magnitudes in a form normalized with respect to the primary coupling would result in zero response for the case of truly coincident coils. In the physical model, the vertical coincident coil configuration requires the placing of the coils at a separation small enough to provide responses that are indistinguishable from the responses of truly coincident coils, while also setting them for zero primary coupling by the use of small axial and radial offsets (DUCKWORTH et al., 1993). By this means only the scattered secondary field is detected (DuCKWORTh et al., 1993; DuCKWORTH, 1994; DuCK- 

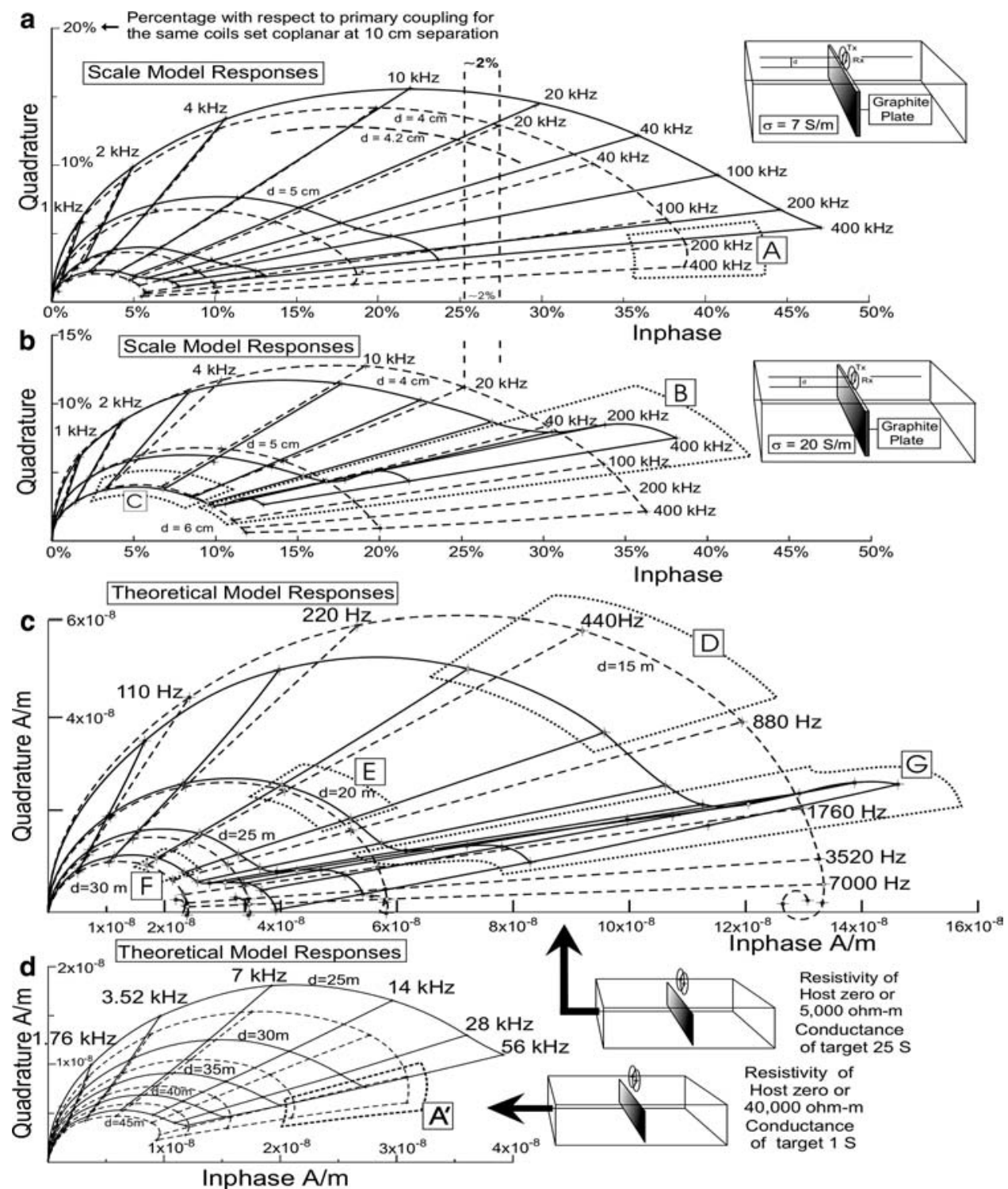

Figure 2

Physical and theoretical model responses of a Vertical Coplanar coil system over a vertical plate. (a) Physical scale model: $819 \mathrm{~S}$ graphite plate in a $7 \mathrm{~S} / \mathrm{m}$ brine. (b) Physical scale model responses for a $819 \mathrm{~S}$ graphite plate in a $20 \mathrm{~S} / \mathrm{m}$ brine. (c) Theoretical model responses for a $25 \mathrm{~S}$ plate in a $0.0002 \mathrm{~S} / \mathrm{m}$ (5000 ohm-m) host. The transition from negative to positive screening as depth increases is notable. (d) Theoretical model responses for a $1 \mathrm{~S}$ plate in a 40,000 ohm-m host. (Note the magnified plotting scale used when compared with Figure 2(c)). The loss of amplitude at high frequency in the free-space responses seen in Figure 2(d) in zone A' is also seen in the Scale Model responses of Figure 2(a) in zone A. In each diagram the conductive host cases are superimposed on the free-space cases, with the free-space cases being represented by dashed contours. Zones B, C, D, E, F, and G are discussed in the main text. 
worth and Krebes, 1997a, b, 1998; and Duckworth and Clement, 2001; Won et al., 1997).

The vertical coplanar coincident coil configuration provides maximum response to a vertical plate when the coils are coplanar with the plate, as shown in Figure 2. In the scale model responses shown in Figures 2(a) and 2(b), the target is the same graphite plate that was used in Figure 1(a). The frequency range is the same as that used in Figure 1(a) and the anomaly magnitudes are presented as percentages of the free-space response obtained with the model coils coplanar at $10 \mathrm{~cm}$ separation. Figures 2(a) and 2(b) present the physical scale model responses for a host conductivity of $7 \mathrm{~S} / \mathrm{m}$ and $20 \mathrm{~S} / \mathrm{m}$, respectively. Again, the responses with the conductive host are superimposed on the free-space responses with the free-space responses represented by dashed contours.

The range of depth values in Figures 2(a) and 2(b) is from $4 \mathrm{~cm}$ to $6 \mathrm{~cm}$ rather than the range from $2 \mathrm{~cm}$ to $5 \mathrm{~cm}$ for the horizontal coplanar scale model responses presented in Figure 1(a). The reason for this use of a greater depth range lies in the difficulty setting the depth of the target with the coils in the vertical coincident configuration. This can be appreciated in the scale model responses shown in Figure 2(a) where the vertical dashed bars indicate that for depths of the order of $4 \mathrm{~cm}$, a change in the depth setting from $4 \mathrm{~cm}$ to $4.2 \mathrm{~cm}$ causes a $2 \%$ difference in the magnitude of the detected field. Thus the fact that the free-space response at $20 \mathrm{kHz}$ in Figures 2(a) and 2(b) differs by approximately 2\% indicates that the nominal $4 \mathrm{~cm}$ depth setting in Figure 2(b) was in fact possibly as much as $4.2 \mathrm{~cm}$. With model coils of $1.5 \mathrm{~cm}$ diameter, set in zero coupling at small separation, a $2 \mathrm{~mm}$ variation in their height above the target was difficult to avoid. For shallower depths, the sensitivity of the system to depth errors becomes greater as can be seen in the marked increase in the separation between the depth contours as depth is reduced. A $2 \mathrm{~mm}$ depth error for the shallower target depth of $2 \mathrm{~cm}$ used in Figure 1 would have resulted in markedly greater discrepancies.

The effect of the less conductive $7 \mathrm{~S} / \mathrm{m}$ host in the scale model responses (Fig. 2(a)), is an overall positive enhancement and anticlockwise rotation of the response with respect to the free-space case for all frequencies and all depths of the target.

By comparison, the scale model results for the more conductive $20 \mathrm{~S} / \mathrm{m}$ host shown in Figure 2(b) display a marked loss of response (or negative enhancement) when the conductive host is present and this is most pronounced for the shallow target depths. In addition, there is an abrupt onset (in the outlined zone B) of a strong positive enhancement at high frequency. It is also notable that in Figure 2(b) the difference between the responses with and without the host becomes less pronounced as target depth increases. For the deepest target, the response below the frequency of onset of strong enhancement is similar to the response in free-space, as can be seen in zone $\mathrm{C}$. In that the differences between the response with the target in free-space and with the conductive host in place are of the order of $2 \%$ for the 
shallow target, these differences might be attributable to the difficulty in setting the depth of the target as decribed above. However, that discussion was concerned with the comparison between the separate experiments that generated the results in Figures 2(a) and 2(b). Those experiments involved a complete re-establishment of the coil and model setup for each experiment and were done at widely separated times. Each setup involved a very difficult setting of the model coils in a decoupled state at small separation. The results with and without the conductive host shown in Figure 2(b) involved no resetting of the coils; it only required the introduction of the conductive host. Thus within Figure 2(b) the depth settings of the coils with respect to the target are the same with and without the conductive host. The unusual effects shown by Figure 2(b) were verified by more than one experiment and these effects reflect a very satisfactory agreement with the responses shown by the theory that appears in Figure 2(c) as discussed later.

It is notable that the relative sizes of this high frequency dispersion with respect to the free-space dispersion, as seen by the physical scale model and by the theoretical model, are in much better agreement for the vertical coplanar coincident coil responses than is the case for the horizontal coplanar coil results in Figures 1(a) and $1(\mathrm{c})$.

In Figure 2(a) a reduction of the free space response of the target that appears at high frequency is highlighted in zone A. This effect did not appear in the data acquired for Figure 2(b) but it did appear in a repeat test of the experiment with the 7 $\mathrm{S} / \mathrm{m}$ host (DUCKWORTH and KREBES 1997a) and it appeared in theoretical modeling for a low conductance target as shown in Figure 2(d) in the zone marked A'. Experience with other coil configurations suggests that at the inductive limit, the response magnitude should stabilize rather than decline. Thus experience might suggest that the effect seen in zone A of Figure 2(a) is an artifact generated by the experimental difficulties that this coil configuration presents. The theoretical responses shown in Figures 2(c) and 2(d) are based upon the concept of coincident dipolar coils of vanishingly small dimensions. By comparison, the physical model coils were distant from that ideal. It is possible that the setup of the coils used in the experiment for Figure 2(a) came closer to the ideal than did the setup in Figure 2(b). The only objective means to determine if a close approximation to the ideal has been achieved is to perform the whole experiment. It appears that if the theoretical results in Figure 2(d) are taken to represent the true situation, i.e., that the high frequency responses should indeed show a loss of amplitude as the inductive limit is approached, then the settings of the coils in Figure 2(b) was not as close to the ideal as was the case in Figure 2(a). However, even if the setup of the coils that gave the data in Figure 2(b) was not ideal, this does not appear to invalidate the results shown in Figure 2(b) because of their notable agreement with the theoretical results shown in Figure 2(c) as discussed below. A tentative explanation for the effect seen in zones A and A' may be found in the geometry of the current induced into a vertical plate by a horizontal dipole that consists of two vortices of opposite polarity 
(Koefoed and Kegge, 1968; Weidelt, 1983). It can be inferred that the relative contributions of these two vortices to the detected response will vary with frequency and possibly also with the relative positions of the physical model coils. The more commonly employed vertical dipole produces only a single current vortex in the plate.

The difference in the effect of the host between Figures 2(a) (where it is dominantly positive) and 2(b) (where it is dominantly negative for shallow target depth) was caused solely by the change in the conductivity of the host and such a change might be dismissed as an artifact of the experimental procedure, given the experimental difficulties discussed above. However the reality of this difference was confirmed by an earlier test of the physical model (DUCKWORTH and KREBES, 1997a) and by the theoretical responses presented in Figures 2.(c) and 2.(d).

The theoretical responses shown in Figure 2.(c) for a vertical thin plate located in a host of $5000 \mathrm{ohm}-\mathrm{m}$ resistivity, display a diminished response (or negative enhancement) in the presence of the host (zone D) that is very similar to that displayed in the scale model results of Figure 2.(b). However, Figure 2(c) displays an additional unexpected effect in that for deep targets the host effect becomes wholly positive (zone F). This transition from negative to positive enhancement with increasing depth can be appreciated by examination of zones $\mathrm{D}, \mathrm{E}$ and $\mathrm{F}$ in Figure 2(c). The theoretical response for a low conductance (1S) target and low conductivity (40000 ohm-m) host shown in Figure 2(d) indicates that when the host is conductive there is overall positive enhancement of the response in a manner very similar to that shown in the scale model responses of Figure 2(a).

For all depth values in Figure 2(c), the high frequency responses show a sharp onset of a dispersion (zone $\mathrm{G}$ ) that can be ascribed to the onset of strong inductive effects in the host and consequent current gathering into the target. This causes a strong positive enhancement of the response for all depth values. This effect appears to be the same detachment effect seen in the physical model results for a high conductivity host in Figure 2(b) (zone B). However the physical model results (Figure 2(b)) show the current gathering effect advancing equally with frequency in zone B for all target depth values, while in zone $\mathrm{G}$ of Figure 2(c) the greater target depths show a clearly greater phase rotation. This difference might be caused by the conductivity of the host in the scale model (Figure 2(b) being lower than would be necessary to match the conductivity used in the theoretical model; the $20 \mathrm{~S} / \mathrm{m}$ conductivity used in Figure 2(b) was the highest value that could be achieved in practice.

An attempt to explain the behavior of the responses shown in Figure 2(c) as target depth decreases for the frequencies below the point at which strong enhancemant begins, might invoke the concept that as the target approaches the surface, the volume above the conductor from which it can gather current from the host becomes smaller. This will reduce the gathered current and the expected enhancement due to that current. This argument would lead to an expectation that the enhancement due to current gathering would weaken as target depth is reduced, 
but not that it would cause the response to become less than the free-space response as is observed in zone D of Figure 2(c). This argument might also apply equally well to the responses seen in the case of separated horizontal coplanar coils in Figure 1, although there the responses are progressively enhanced as target depth is reduced so that in that case this argument appears to fail. Thus the observed effect in which the conductive host response is less than the free-space response (zone D, Fig. 2(c)) appears to be caused by an additional effect. This additional effect might be propagation losses within the host, nonetheless this effect would get stronger the deeper the target lay within the conductive host and would be minimal for a target lying just below the surface of the host. Thus it seems that propagation losses could not cause the increasing loss as the target depth declined from zone $\mathrm{E}$ to zone $\mathrm{D}$ of Figure 2(c). A third possible cause may lie in the relative geometry of current vortices induced into the target and the host. The behavior shown in zone D suggests that the current vortex inducted into the shallow depth target is in opposite phase with respect to the predominant current vortex near the surface of the host, so that the gathering of an opposite phase current from the host would diminish the current within the target. It appears that current flow at greater depth within the host may have the same phase as the current vortex within the plate target, and that when this deep current is gathered into the target it produces the expected enhancement. Investigation of the relative contributions from these various possible causes of the effects seen in Figure 2 could possibly be accomplished in either physical or theoretical modeling however that is an investigation beyond the scope of the present study.

In the high frequency zone $G$ the enhancement effect of gathered current is able to overwhelm any other effects for any depth of the target and this suggests that if opposite polarity of induced currents within the host and a shallow target explain zone $\mathrm{D}$ then at higher frequencies these currents have the same phase.

In practical operation, the sensitivity of coincident coils to any conductive material close to the coils will cause such a system to respond strongly to geologically uninteresting near surface conductors in much the same manner as magnetometers respond most strongly to near surface magnetic structures. The noise that results, should be amenable to the same forms of treatment by spatial filtering that are used in separating magnetic responses that come from shallow and deep targets.

\section{Fixed Loop Responses}

Anomaly index diagrams have seen little use in the interpretation of fixed loop data nonetheless they are useful in demonstrating the dramatic effect of current gathering in responses provided by fixed loop systems. Figure 3(a) shows scale model responses obtained with a $1 \mathrm{~m}$ square loop transmitter located $60 \mathrm{~cm}$ from and parallel to the same $819 \mathrm{~S}$ graphite plate used in the moving source data shown in Figures 1(a), 2(a) and 2(b). These data are presented in dimensionless form 

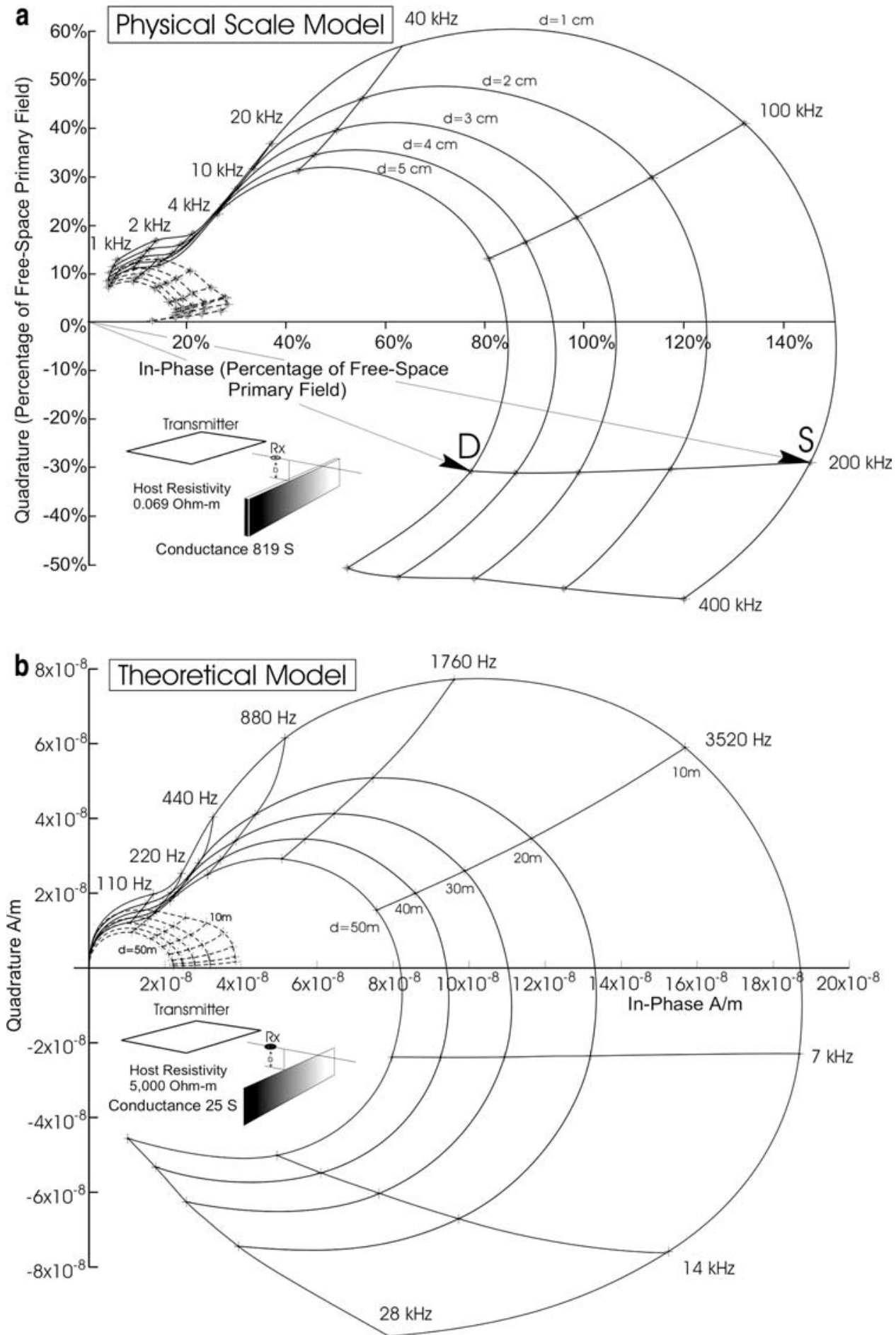
normalized with respect to the signal recorded due to the transmitter alone in freespace for the receiver located at a point that would be directly over the target if the target was present. The responses shown in Figure 3(b) were generated by the thin plate algorithm in the EMIGMA software for a $1 \mathrm{~km}$ square transmitter located $500 \mathrm{~m}$ from a $25 \mathrm{~S}$ vertical plate lying parallel to the near side of the loop. These results are presented in absolute secondary field magnitudes.

In both sets of data the free-space responses of the target are again presented as dashed contours.

The frequency range covered in the physical modeling is from $1 \mathrm{kHz}$ to $400 \mathrm{kHz}$ while in the theoretical responses the range is from $110 \mathrm{~Hz}$ to $28,000 \mathrm{~Hz}$. Depth range in the physical model responses is from $1 \mathrm{~cm}$ to $5 \mathrm{~cm}$ and in the theoretical model from $10 \mathrm{~m}$ to $50 \mathrm{~m}$. The conductivity of the host used in the physical model was $9.93 \mathrm{~S} / \mathrm{m}$ and in the theoretical model $0.0002 \mathrm{~S} / \mathrm{m}$ (i.e., $5000 \mathrm{ohm}-\mathrm{m}$ resistivity).

The outstanding feature in both data sets is the dramatic enhancement of the response when the conductive host is in place and detachment of that response from the free-space response. For the $1 \mathrm{~cm}$ scale model target depth in Figure 3(a), the response with the conductive host in place is approximately $530 \%$ stronger than the free-space response in the frequency range from $100 \mathrm{kHz}$ to $200 \mathrm{kHz}$. Correspondingly the $7 \mathrm{kHz}$ theoretical response in Figure 3(b) for the $10 \mathrm{~m}$ target depth with the conductive host in place, is approximately $475 \%$ stronger. At the $5 \mathrm{~cm}$ scale model depth in Figure 3(a), the response with the conductive host is $490 \%$ stronger than the free-space response and in Figure 3(b) for the $50 \mathrm{~m}$ depth in the theoretical model the conductive host causes a 370\% stronger response. Thus for greater target depth there is less enhancement in both the scale model and theoretical responses. This confirms the decline of the current gathering effect in fixed loop responses as target depth increases, that was discussed in detail by DUCKWORTH et al. (2001). It is notable that while the theoretical and physical model responses differ in detail, their overall agreement in terms of the size of the enhanced response with the conductive host in place versus the free-space response is notably better for these fixed loop responses than was the case for the moving source responses in Figure 1. In the fixed loop physical model work the receiver coil was effectievly smaller with respect to the overall model than was the case in the moving source horizontal coplanar coil system used in Figure 1. This suggests that the out-of-scale size of the coils in the moving source experiment may have partially caused the difference in results seen in that case.

\section{Figure 3}

Physical scale model and theoretical model responses for a vertical thin plate detected by means of a fixed loop system. (a) Physical scale model responses for an $819 \mathrm{~S}$ graphite plate in a host of $0.069 \mathrm{ohm}-\mathrm{m}$ resistivity for a range of depth values. (b) Theoretical model responses for a $25 \mathrm{~S}$ plate in a $5000 \mathrm{ohm}-\mathrm{m}$ host for a range of depth values. In each diagram the conductive host cases are superimposed on the freespace cases, with the free-space cases being represented by dashed contours. 
In Figure 3 the onset of the strong current gathering effect occurs at lower frequency in these fixed loop results than in the moving source results shown in Figure 1. Separate results (not shown) derived by theoretical modeling in which the size of the transmitter was reduced, created both a smaller enhancement and a higher frequency of onset.

The phase inversion shown by both sets of results in Figure 3 is unlikely to be seen in full scale field data as it occurs at a frequency of $7000 \mathrm{~Hz}$ in Figure 3(b) and that is a frequency that has seldom been used in fixed loop field surveys. However surveys conducted at the Night Hawk test range (PITCHER, 1985) came close to such an inversion at a frequency of $2025 \mathrm{~Hz}$ (DUCKWORTH and O'NeILL, 1992). It is also notable that both modeling approaches indicate a greater phase rotation for the deeper target responses (as indicated by the vectors S and D in Figure 3(a)) which is

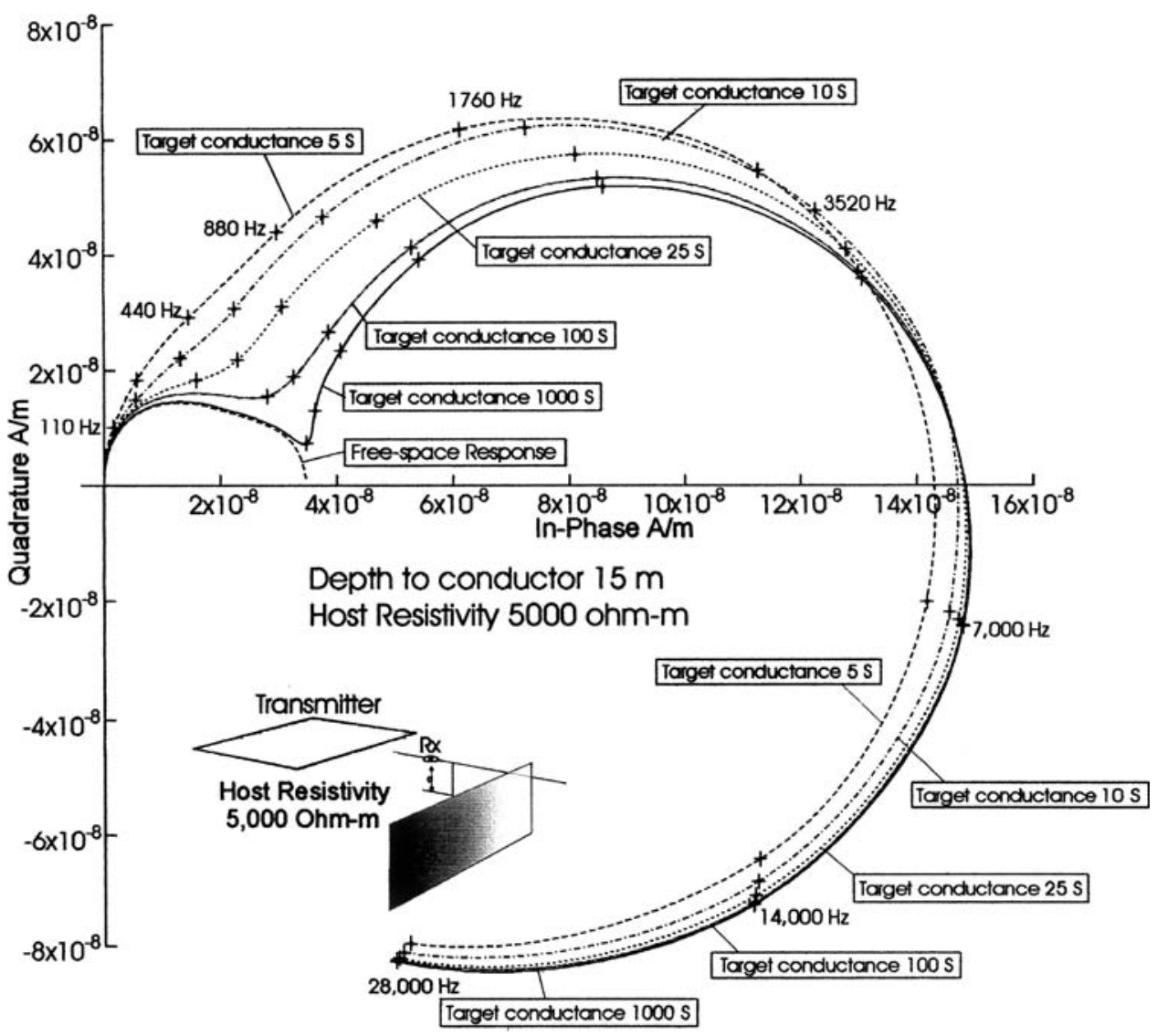

Figure 4

Theoretical model responses for a vertical thin plate detected by means of a fixed loop system. The target is at a single depth of $15 \mathrm{~m}$ located in a $5000 \mathrm{ohm}-\mathrm{m}$ host. For frequencies above $3520 \mathrm{~Hz}$ the responses of conductors of widely different conductance become almost identical. 
to be expected when considering the phase rotation of fields passing to and from the target in terms of the path length of propagation through the host.

Figure 4 shows the variation of current gathering with changing target conductance in a target fixed at $15 \mathrm{~m}$ depth. These are theory-generated responses for targets of $5 \mathrm{~S}, 10 \mathrm{~S}, 25 \mathrm{~S}, 100 \mathrm{~S}$ and $1000 \mathrm{~S}$ conductance in a 5000 ohm-m host. For frequencies below $3520 \mathrm{~Hz}$, the responses for the $5 \mathrm{~S}$ to $100 \mathrm{~S}$ range are well separated so that resolution of target parameters appears to be possible in this frequency range. Albeit the conductance values derived from such data would clearly be poorly related to the actual conductance of the target. At higher frequencies the responses tend to become almost identical; much as was the case in the moving source responses of Figures 1(b), and 1(c).

The considerably stronger current gathering effects displayed by the fixed loop system than are displayed by moving source responses, and appear to be caused by the much larger volume of the host into which current is induced by the fixed loop transmitter. The strength of current gathering exhibited in Figure 3(b) for a $5000 \mathrm{ohm}-\mathrm{m}$ host, indicates that fixed loop systems gain the great majority of their advantage in generating detectable secondary fields because of the current gathering effect. This contrasts with the normal justification of the use of fixed loop systems which is that their potential for greater depth of exploration is based on the greater transmitter moment and lower geometric losses they offer when compared to moving source systems.

\section{Conclusions}

The results provided by a thin plate theoretical algorithm are in good agreement with results generated by physical scale modeling. This comparison of scale model and theoretical modeling demonstrates that the current gathering phenomenon may cause effects that are not easily recognized and that are in some cases counter to expectation.

The two modeling approaches are in notable agreement in the simulation of the three exploration systems concerning the onset of a strong current gathering dispersion at high frequencies. Both approaches demonstrate that the frequency of onset of this strong current gathering effect will be lower for fixed loop systems than for moving source systems. Both methods also agree on an unexpected attenuation of the response provided by vertical coincident coils operating at low frequencies over shallow depth targets. For the explanation of this effect to be fully understood, additional modeling will be required.

The relative magnitude of the current gathering effect with respect to the response of the target in free-space is in good agreement in the physical modeling and theoretical approaches for the fixed loop and vertical coincident coil responses but notably not in agreement in the case of separated horizontal coplanar coils. The 
dramatic enhancement of fixed loop responses, and their phase inversion at high frequencies indicates that fixed loop methods derive most of their response to mineral targets from the current gathering phenomenon.

Both modeling methods indicate that the use of higher transmitter frequencies will cause the responses generated by moving source horizontal coplanar coils operated over targets of widely different conductance to become almost indentical because those high frequency responses are dominated by the current gathered from the host.

\section{Acknowledgements}

This work was supported by a Research Grant from the Natural Science and Engineering Research Council of Canada.

\section{REFERENCES}

Duckworth, K., Nichols, T.D., and Krebes, E.S. (2001), Examination of the Relative Influence of Current Gathering on Fixed Loop and Moving Source Electromagnetic Surveys, Geophys. 66, 10591066.

Duckworth, K., and Clement, B. (2001), Inductive Interaction between Closely Spaced Steeply Dipping Tabular Conductors Located in a Resistive Host, Geophys. 66, 1052-1058.

Duckworth, K., and Krebes, E.S. (1998), Theoretical Response of Vertical Coincident and Horizontal Coplanar Coils when Operated Over Steeply Dipping Conductors Located below a Conductive Overburden, Geophys. 63, 841-855.

Duckworth, K., and Krebes, E.S. (1997a), The Response of Steeply Dipping Tabular Conductors Located in Conductive Host Rocks when Detected by Horizontal Coplanar Coil and Vertical Coincident Coil Electromagnetic Exploration Systems, Can. J. Expl. Geophys. 33, 55-65.

Duckworth, K., and Krebes, E.S. (1997b), Depth Sounding by Means of Coincident Coil Frequencydomain Electromagnetic System, Geophys. 62, 49-55.

Duckworth, K. (1994), The Response of a Coincident-coil Electromagnetic Prospecting System to Targets Located below Conductive Overburden, Can. J. Expl. Geophys. 30, 1-10.

Duckworth, K., Krebes, E.S., Juigalli, J., Rogozinski, A., and Calvert, H.T. (1993), A Coincident Coil Frequency-domain Electromagnetic Prospecting System, Can. J. Expl. Geophys. 29, 411-418.

Duckworth, K., and O'Neill, D. (1992), Comparison of Scale Model Results with Field Surveys Conducted over the Night Hawk Test Range Using Fixed-loop and Moving Source Electromagnetic Systems, Can. J. Expl. Geophys. 28, 1-5.

Duckworth, K., and O'Neill, D. (1989), Turam Responses in a Scale Model Conductive Environment for Single and Paired Conductors, Can. J. Expl. Geophys. 25, 115-137.

Farquharson, C.G., Duckworth, K., and Oldenburg, D.W., Comparison of Integral Equation and Physical Scale Modelling of the Electromagnetic Response of Models with Large Conductivity Contrasts, CGU Annual Conference, Banff, 2003.

Frischknecht ,F.C., Labson, V.F., Spies, B.R., and Anderson, W.L. (1991), Profiling Methods Using Small Sources. In Electromagnetic Methods in Applied Geophysics, Vol. 2, Application, Part A (ed, Nabighian, M.N.) 105-252. 
Guptasarma, D., and Maru., V.M. (1971), A Study of Some Effects of a Conducting Host Rock with a new Modeling Apparatus, Geophys. 36, 166-183.

Hanneson, J.E., and West, G.F. (1984), The Horizontal Loop Electromagnetic Response of a Thin Plate in a Conductive Earth: Part II - Computational Results and Examples, Geophys. 49, 421-432.

Hedstrom, H., and Parasnis, D.S. (1958), Some Model Experiments Relating to Electromagnetic Prospecting with Special Reference to Airborne Work, Geophys. Prosp. 6, 322-341

Koefoed, O., and Kegge, G. (1968), The Electrical Current Pattern Induced by an Oscillating Magnetic Dipole in a Semi-infinite Thin Plate of Infinitesimal Resistivity, Geophys. Prosp. 16, 144-158

Lajoie, J.J., and West, G.F. (1976), The Electromagnetic Response of a Conductive Inhomogeneity in a Layered Earth, Geophys. 41, 1133-1156.

Lowrie, W., and West,G.F. (1965), The Effect of Conducting Overburden on Electromagnetic Prospecting Measurements, Geophys. 30, 624-632.

Nair, M.R., Biswas, S.K., and Mazumdar, K. (1968), Experimental Studies on the Electromagnetic Response of Tilted Conducting Half-planes to a Horizontal Loop Prospecting System, Geoexploration 6, 207-244.

Pitcher, D.H. (1985), Night Hawk geophysical test range results, District of Cochrane. In (Wood,J., White, O.L., Barlow, R.B., and Colvine, A.C., eds.) Summary of Field Work: Ont. Geol. Surv., Misc. Paper 126, pp.170-177.

StrangWay, D.W. (1966), Electromagnetic scale modeling. In Methods and Techniques, in Geophys. 2, (ed., Runcorn, S.K.) (Interscience Publishers, John Wiley and Sons, 1996).

Walker, P.W., and West, G.F. (1991), A Robust Integral Equation Solution for Electromagnetic Scattering by a Thin Plate in Conductive Media, Geophys. 56, 1140-1152.

Weidelt, P. (1983), The Harmonic and Transient Response of a Thin Dipping Dyke, Geophys. 48, 934-953.

Won, I.J., Keiswetter, D., Hanson, D., Novikova, E., and Hall, T. (1997), GEM-3: A Monostatic Broadband Electromagnetic Induction Sensor, J. Environ. Eng. Geophys. 2, (1) 53-64.

(Received May 27, 2005; accepted February 9, 2005)

(20) To access this journal online:
http://www.birkhauser.ch 